\title{
Solving Visual Madlibs with Multiple Cues
}

\author{
Tatiana Tommasi ${ }^{1}$ \\ ttommasi@cs.unc.edu \\ Arun Mallya ${ }^{2}$ \\ amallya2@illinois.edu \\ Bryan Plummer ${ }^{2}$ \\ bplumme2@illinois.edu \\ Svetlana Lazebnik ${ }^{2}$ \\ slazebni@illinois.edu \\ Alexander C. Berg ${ }^{1}$ \\ aberg@cs.unc.edu \\ Tamara L. Berg ${ }^{1}$ \\ tlberg@cs.unc.edu
}

${ }^{1}$ University of North Carolina at Chapel Hill, (NC) USA

2 University of Illinois at Urbana-Champaign, (IL) USA
This paper focuses on answering multiple choice questions from the Visual Madlibs dataset [2] which was created by asking people to write fill-in-the-blank descriptions about persons (action, attribute, location), objects (affordance, attribute, location), and high-level concepts as future and past events.

We posit that in order to truly understand an image and answer questions about it, it is necessary to leverage rich and detailed global and local information. To explore this assertion, we represent the images by using $\mathrm{CNN}$ architectures trained on task-specific sources to recognize more than 200 scenes, 900 actions and 300 attributes (see Fig. 1). We extract the features both from the whole image and from regions selected to best match people and objects mentioned in the answers. We project both the visual and textual information in a joint CCAembedding space [1] and at test time, we select the putative answer which obtains the highest cosine similarity with the image features. Finally we integrate multiple cues, through lowlevel visual feature stacking and high-level CCA score combinations. Our results show a significant improvement over the previous state of the art (see Tab. 1), and indicate that answering different question types benefits from examining a variety of image cues and carefully choosing informative image sub-regions.

[1] Y. Gong, Q. Ke, M. Isard, and S. Lazebnik. A multi-view embedding space for modeling internet images, tags, and their semantics. IJCV, 2014.

[2] L. Yu, E. Park, A. C. Berg, and T. L. Berg. Visual Madlibs: Fill in the blank Image Generation and Question Answering. In ICCV, 2015.
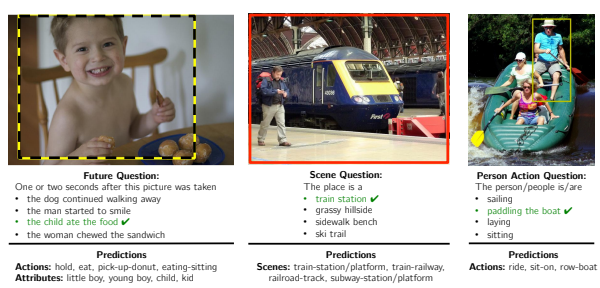

Figure 1: Our method uses multiple deep networks trained on external knowledge sources to predict action, attribute, scene, and other diverse features from specific regions in the image. A CCA model trained on these features allows to score the putative answers and select the correct one for different different types of questions.

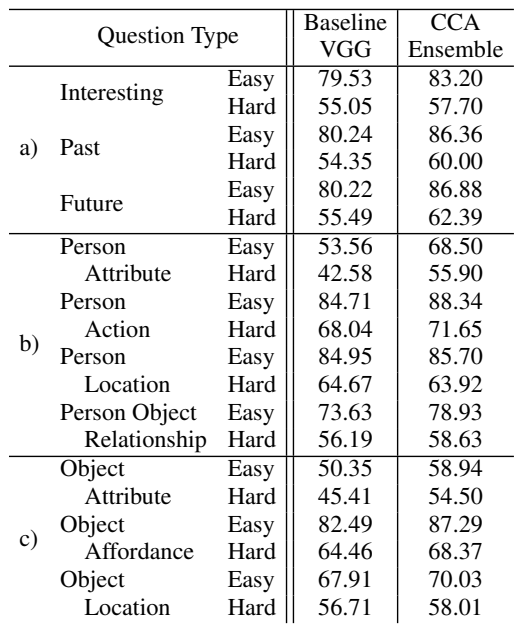

Table 1: Improvement in accuracy by combining CCA scores from multiple cues. 